\title{
The Importance of a Conchal Bowl Element in the Fabrication of a Three-Dimensional Framework in Total Auricular Reconstruction
}

\author{
Young Soo Kim \\ Bona Microtia and Aesthetic Ear Surgery Clinic, Seoul, Korea
}

Background To construct a sophisticated three-dimensional framework, numerous modifications have been reported in the literature. However, most surgeons have paid little attention to the anatomical configuration of the concha and more to its deepness and hollowness, leading to unsatisfactory outcomes.

Methods For a configuration of the concha that is definitely anatomical, the author further developed and employed the conchal bowl element, which has been used by several surgeons although the results have not been published elsewhere. The author constructed the conchal bowl element in one of three patterns according to the amount of available cartilages: one block, two-pieces, or a cymba bowl element only. A total of 20 patients underwent auricular reconstruction using a costal cartilage framework between 2009 and 2012. The 8 earliest reconstructions were performed without a conchal bowl element and the latter 12 with a conchal bowl element. The patients were followed up for more than 1 year. The aesthetic results were scored by evaluating characteristics involving the stability of the crus helicis, the conchal definition, and the smoothness of the helical curve.

Results The ears reconstructed early without a conchal bowl element showed a shallow and one or two incompletely separated concha with an obliterated cymba conchal space. They also did not have a realistic or smooth curve of the helix because of an unstable crus helicis. However, ears reconstructed later with the concha bowl element showed a definite crus helicis, deep cymba conchal space, and smooth helical curve.

Conclusions The construction of the conchal bowl element is simple, not time-consuming procedure. It is suggested that the conchal bowl element must be constructed and attached to the main framework for natural configuration of the reconstructed ear.

Keywords Ear, external / Hyaline cartilage / Reconstructive surgical procedures
Correspondence: Young Soo Kim Bona Microtia and Aesthetic Ear Surgery Clinic, 208th, 3rd floor, Mujin building, Dosan-daero, Gangnam-gu, Seoul 135-816, Korea Tel: +82-2-593-0133

Fax: +82-2-593-0233

E-mail:yskim1126@hotmail.com

Received: 28 Nov 2012 • Revised: 9 Mar 2013 - Accepted: 27 Mar 2013

pISSN: 2234-6163・ elSSN: 2234-6171 • http://dx.doi.org/10.5999/aps.2013.40.3.192 • Arch Plast Surg 2013;40:192-197

This article was presented at the 5 th International Congress on Auricular Reconstruction, March 11-13, 2012 in Sydney, Australia and the 70th Congress of the Korean Society of Plastic and Reconstructive Surgeons, November 9-11, 2012 in Seoul, Korea.

No potential conflict of interest relevant to this article was reported.

\section{INTRODUCTION}

Total ear reconstruction is a complex and challenging procedure for plastic surgeons. Fabrication of a three-dimensional frame- work with delicate anatomical landmarks is a critical factor in achieving a successful result. Construction of a sophisticated framework is not easy and has a long learning curve. Numerous modifications of the cartilage framework have been introduced 
for realization of a natural-looking auricle. However, most of the fabricated frameworks have incomplete or missing structures, especially in the conchal area. The concha is shallow and has one or two incompletely separated units with an obliterated cymba conchal space. The reconstructed ear does not have a realistic and smooth curve of the helix because most cartilage frameworks do not have a conchal bowl element but empty space divided by a partial or complete crus helicis. Therefore, the reconstructed ear will be unsatisfactory. Anatomically, the concha has not only a complete crus helicis but also two major components, the cymba and cavum concha, with similar depth to each other.

The concha is one of the key elements in three-dimensional auricular reconstruction. In this article, the author presents the importance of the conchal bowl element in the fabrication of the framework for a three-dimensional appearance and reports on the outcomes with or without a conchal bowl element. Applications to various clinical situations are also suggested.

\section{METHODS}

The fabrication of a costal cartilage framework followed the method introduced by Nagata [1-5] with some modifications. The sixth through ninth costal cartilages were harvested from the side ipsilateral to the reconstructed ear through a transverse 4 to $5 \mathrm{~cm}$ incision into the mid-chest, with the whole perichondrium intact. The base frame was constructed from the sixth and seventh costal cartilages. The eighth costal cartilage was used for construction of the helix and crus helicis unit. For the construction of the superior and inferior crus and antihelix unit, the ninth costal cartilage was used. The remaining cartilages were used for construction of the tragal complex and conchal bowel element. After the fabrication of the costal cartilage framework was completed, a number of small pieces of costal cartilage were cut into 2 to $3 \mathrm{~mm}$ blocks and returned into the perichondrial pocket, following Kawanabe and Nagata $[6,7]$.

\section{Fabrication of the framework}

In most young people, a synchondrial portion between the 6th and 7 th costal cartilages is loose and sometimes unstable. To construct a stable base framework, the author always removed the syncondrial portion, rather than reinforcing it with wire [8] or supplementing it with an additional thin sheet [9], for prevention of instability and step deformity, which could have developed later. After removal of the synchondrosis, the 6th and 7 th cartilages were carved as a base frame and securely fixed with 38-gauge wires at multiple points. However, for patients in old age, the base frame was constructed with the 6 th and 7 th car- tilages en bloc. Other structures such as the helix, antihelix, and tragal complex were constructed with the 8th and 9th, any other remaining cartilages. Each constructed structure was placed on the top of the base frame and firmly fixed with 38-gauge wires. In the early period, the fabrication of the framework was completed at this step, but the results were unsatisfactory in some ways. First, the crus helicis was sometimes unstable, so a realistic and smooth curve of the helix was not obtained. Second, the depth of the conchal bowl was shallow, and the reconstructed ear showed one or even two separated conchae with an obliterated cymba conchal space. This resulted in a non-anatomical and two-dimensional appearance.

Therefore, in the latter cases, the conchal bowl element, which is used by several surgeons, was attached to the fabricated main framework for a three-dimensional appearance.

\section{Fabrication of the conchal bowl element}

The roles of the conchal bowl element are to stabilize the crus helicis and then to achieve a smooth, natural helical curve. At the same time, it converts a two-dimensional structure into a three-dimensional one. The reconstructed ear shows a deep, hollow, and definitely separate concha, and the cymba and cavum conchae have a similar level of depth to each other. The author constructed the conchal bowl element in one of three patterns according to the amount of available costal cartilages. Among the three patterns, the one-block pattern and the pattern with two-pieces of conchal bowl have already been introduced by Nagata. However, Nagata's conchal bowl element has some limitations, which cause a step deformity in the cavum concha area and relatively high crus helicis. Therefore, the author modified Nagata's conchal bowl element at some points, and if the remaining cartilages were not sufficient to construct the one or two pieces of the conchal bowl element, the author fabricated only the cymba bowl element to achieve similar effects.

\section{Modifications of the conchal bowl element}

To overcome some limitations of Nagata's conchal bowl element, the author has introduced modifications in the fabrication of the conchal bowl element (Fig. 1). First, the crus helical portion in the conchal bowl element is carved extremely thinly, except a portion of the ascending helical root. Therefore, the height of the complex with the crus helicis and conchal bowl element is slightly higher than the cymba crater at the conchal floor level. Second, to avoid the step deformity caused by the cavum component in Nagata's conchal bowl element, the author constructed a cavum component of minimal size in the fabrication of the conchal bowl element. Two modifications in the construction of the conchal bowl element allow the recon- 


\section{Fig. 1. Modifications of the conchal bowl element}

One-block conchal bowl element (anterior view). Modifiction 1 is that the crus helical portion in the conchal bowl element is carved extremely thinly except a portion of the ascending helical root. Therefore, the height of the complex with the crus helicis and conchal bowl element is slightly higher than the cymba crater at the conchal floor level. Modification 2 is a minimally sized cavum component in the conchal bowl element in order to avoid the step deformity in the concha.

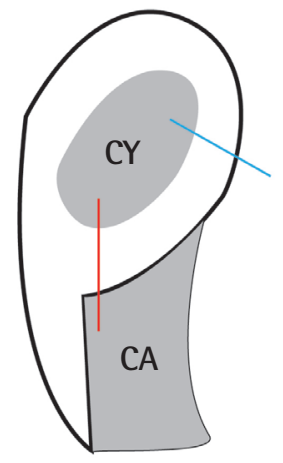

\section{Modification 1.}

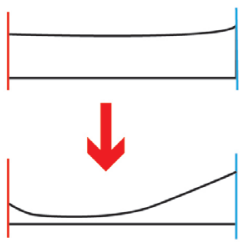

Modification 2.

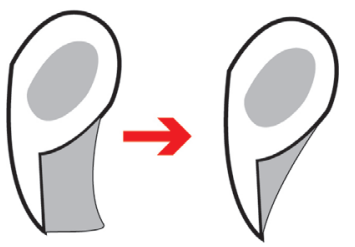

: Cymba (CY) and cavum (CA) crater

structed ear to show natural and realistic contours in the concha and helical root area.

\section{The patterns of the conchal bowl element}

According to the amount of available costal cartilages, the author constructed the conchal element in one of the following three patterns:

One-block conchal bowl element (Fig. 2): If the amount of remaining costal cartilages is sufficient to construct a one-block conchal bowl, a block is carved in one piece for the conchal bowl. The conchal bowl has two concave craters (corresponding to the cymba and cavum conchae) and tapered peripherally.

Two-pieces conchal bowl element (Fig. 3): Two conchal bowls are constructed separately when the remaining cartilages are inadequate for constructing a one-block conchal bowl.

Only the cymba bowl element (Fig. 4): If the available costal cartilages are not sufficient to fabricate a one- or two-pieces conchal bowl element, the author constructed only the cymba bowl element. Though the framework has less stability than it would with the one- or two-pieces element, the purpose of the conchal bowl element, that is, to stabilize the crus helicis and then to realize a smooth and natural helical curve, can be achieved by the cymba bowl element alone.

The fabrication of the conchal bowl element is the last step in

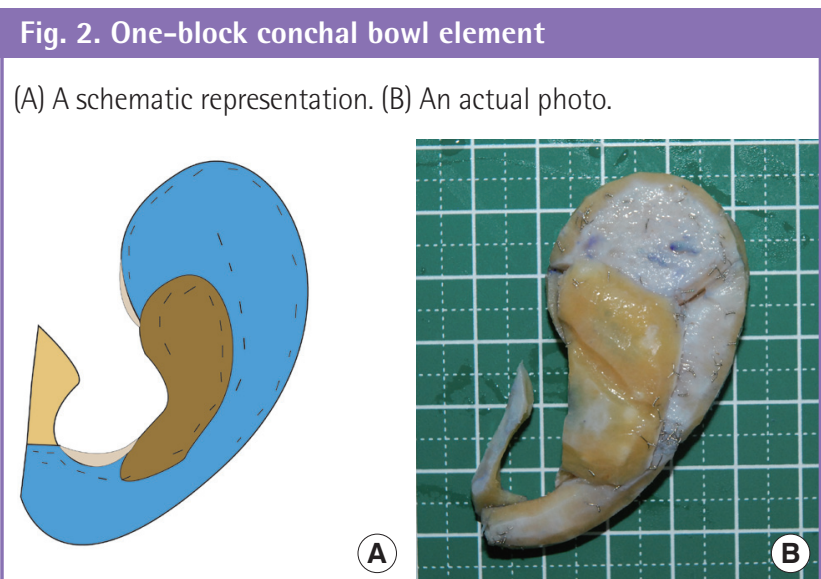

Fig. 3. Two-pieces conchal bowl element

(A) A schematic representation. (B) An actual photo.

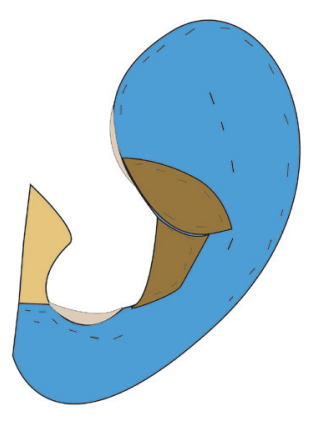

(A)

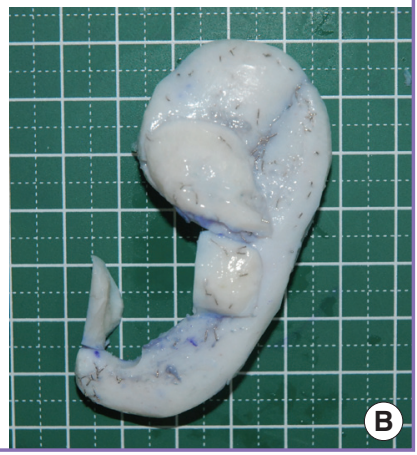

Fig. 4. Cymba bowl only

(A) A schematic representation. (B) An actual photo.

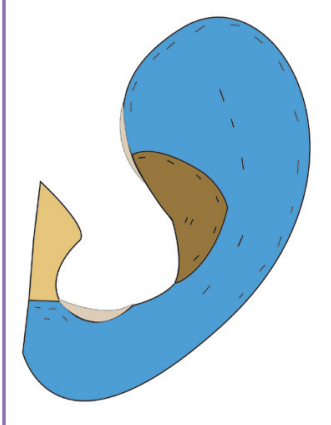

(A)

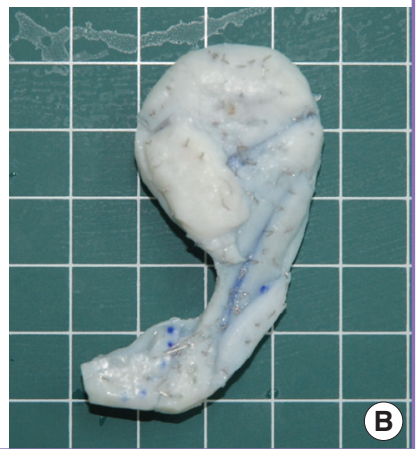

the construction of the auricular framework. After the fabricated framework is inserted into the skin pocket, it is important to check whether skin tension exists or not. If excess skin tension exists, it is necessary to adjust the height of the conchal bowl element.

\section{RESULTS}

A total of 20 patients underwent auricular reconstruction us- 
ing autogenous costal cartilage between 2009 and 2012. The ages of the patients ranged from 12 to 49 years (mean age, 18.1 years). Fifteen patients were male and 5 were female. Nine cases had the lobular type of microtia, 6 had the concha type, 2 had the small concha type, and 2 the atypical, while 1 was a clinical anotia case. The earliest 8 patients underwent ear reconstruction using a costal cartilage framework without a conchal bowl element and the latter 12 patients with a conchal bowl element. Among the patients with the conchal bowl element, 5 patients had one block, 3 patients had a two-pieces element, and 4 patients had the cymba bowl only. Among those with the conchal bowl element, the distribution of the microtia type was as follows: 4 were of the concha type, 4 of the lobular type, 2 of the small concha type, and 2 of the atypical type.

The patients were followed up for more than 1 year. The aesthetic results were assessed by independent observers, using

Fig. 5. The reconstructed ear without the conchal bowl element

Examples of the reconstructed ear without the conchal bowl element. (A) The result 13 months after surgery in a 13-year-old boy with right lobule type microtia. Note the contracted crus helicis, incompletely defined cymba conchal space, and uneven helical curve. (B) The result 14 months after operation in a 12-year-old boy with right concha type microtia. There are discontinuities between the crus helicis and ascending helix.
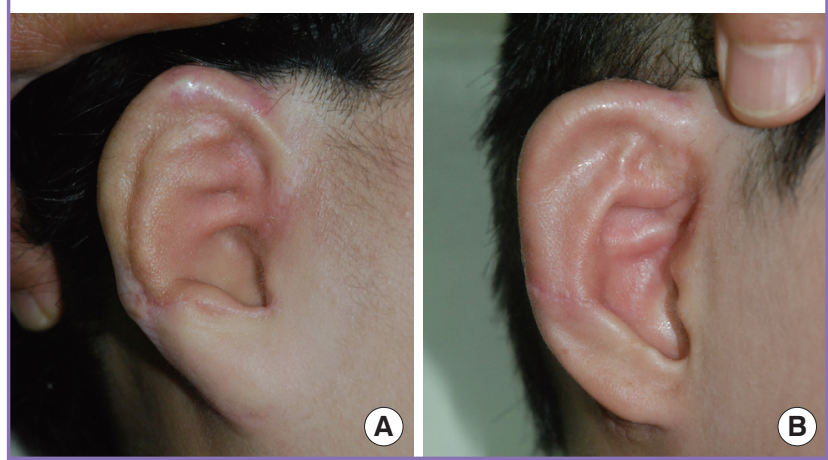

postoperative photographs and scored as 0 (poor), 1 (fair), 2 (good), or 3 (excellent). The categories involved the stability of the crus helicis, the conchal definition involving the depth of the cymba concha, and the smoothness of the helical curve. The aesthetic outcome was recorded as a sum of the scores for each category. The ears reconstructed early, without the conchal bowl element, showed a shallow and one or two incompletely separated concha with the cymba conchal space obliterated (Fig. 5). The aesthetic results ranged from 2 to 5 points (mean 3 points). However, the ears reconstructed later, with the concha bowl element, showed a definite crus helicis, deep cymba conchal space, and smooth helical curve (Fig. 6), and their scores were from 7 to 9 points (mean, 8 points). Therefore, most of the patients who had undergone surgery more recently in this series were more satisfied than the early patients because of the smooth helical curve and definite convolutions of the auricle. The following cases represent the reconstructed ear with and without the conchal bowl element.

\section{Case reports}

Case 1 (framework without conchal bowl element)

A 24-year-old woman presented with atypical-type microtia. The

\section{Fig. 6. The reconstructed ear with the conchal bowl element}

Examples of the reconstructed ear with a conchal bowl element. View 12 months after the first-stage operation in a 16-year-old girl with right small concha type microtia. A smooth helical curve and definite cymba conchal space can be noted.

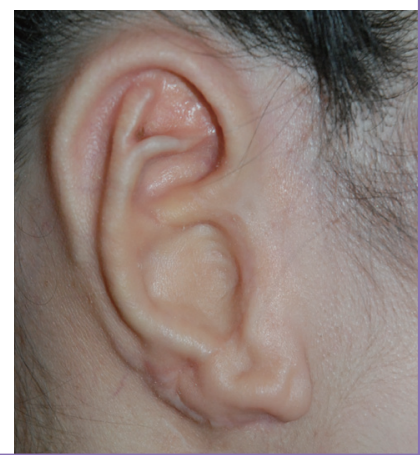

\section{Fig. 7. Representative case 1}

Framework without conchal bowl element. A 24-year-old women with right atypical type microtia. (A) Preoperative view. (B) Fabricated costal cartilage framework. (C) View 15 months postoperatively. Note the relatively acceptable result, but the shallow depth of the cymba compared to the cavum concha.
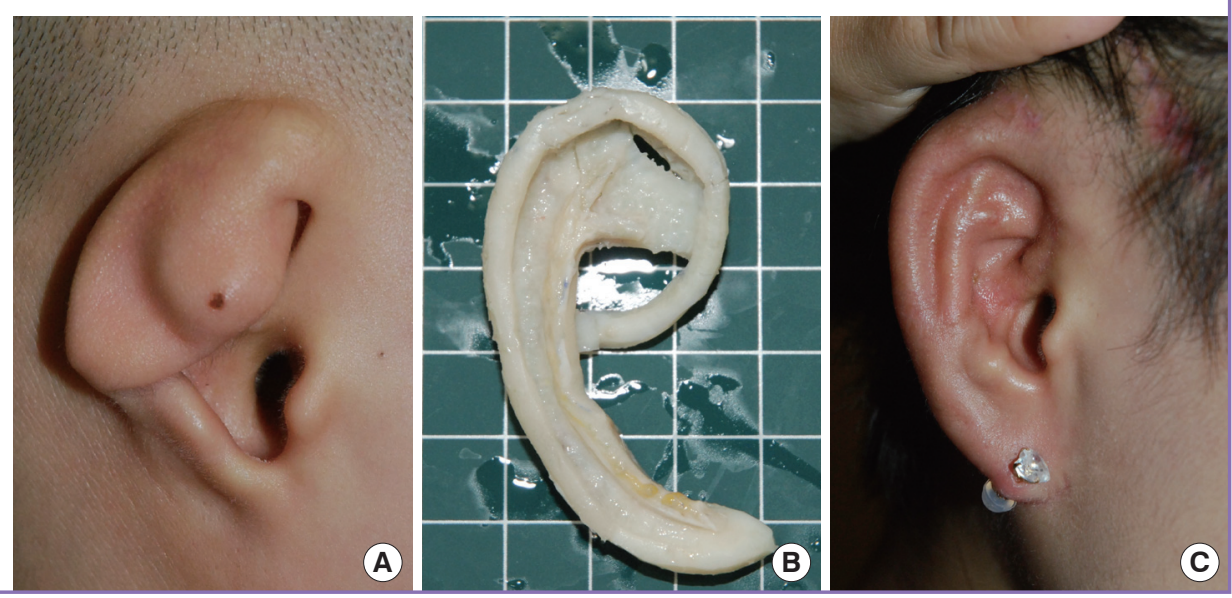
Fig. 8. Representative case 2

Framework with conchal bowl element. A 49-year-old man with left small concha type microtia. (A) Preoperative view. (B) Fabricated costal cartilage framework with one-block concha bowl element. (C) Thirteen months postoperatively. Note a definite crus helicis, conchal space with a deep cymba, and a natural helical curve.
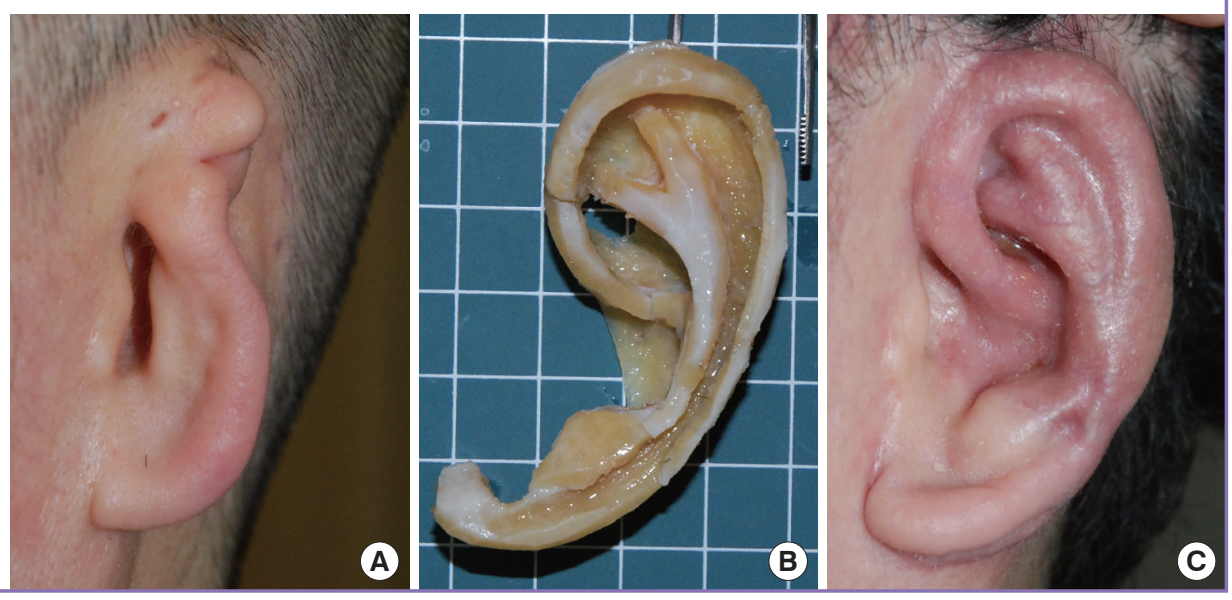

fabricated framework lacked a conchal bowl element. Therefore, the cymba concha was left as an empty space, as most surgeons would do. The reconstructed ear 15 months after the operation showed a relatively acceptable outcome, but the shallow depth of the cymba compared to cavum concha should be noted (Fig. 7). Its aesthetic score was recorded as 5 points.

\section{Case 2 (framework with conchal bowl element)}

A 49-year-old man presented with small concha type microtiaA three-dimensional costal cartilage framework was fabricated, in which a one-block conchal bowl element was attached to the main frame. The reconstructed auricle 13 months after the operation showed a definite crus helicis, a conchal space with deep cymba, and a natural helical curve (Fig. 8). Its aesthetic score was recorded as 9 points.

\section{DISCUSSION}

The fabrication of a sophisticated auricular framework is a prerequisite for a successful reconstruction of the ear. Accordingly, numerous modifications in the construction of the costal cartilage framework have been introduced over the past years in the literature. However, most cartilage frameworks are incomplete or insufficient for re-creation of the three-dimensional structures of the ear, especially in the conchal area. In the fabrication of a framework, most surgeons, including Tanzer [10], Brent [1113], Osorno [14], and Firmin [15], have paid little attention to the anatomical configuration of the concha and more to its deepness and hollowness. The fabricated framework does not have a definite conchal bowl element but empty space divided by a partial or complete crus helicis. The reconstructed ear also does not have a realistic and smooth curve of the helix due to an unstable crus helicis. This may be caused by the herniation of tissues into the empty conchal space and contracted crus helicis during the healing period. The lack of definition, especially in the cymba area, cannot be effectively improved by corticosteroid injection because of a narrow and shallow cymba area due to the contracted crus helicis. Therefore, the reconstructed ear will be unsatisfactory.

The concha is one of the key landmark in the three-dimensional architecture of the ear. Tolleth [16] described the concha is a shallow, almost triangular cuplike structure with a depth of 1.5 $\mathrm{cm}$. It is divided by the root of the helix into a superior cymba conchae and larger inferior cavum conchae.

For a definitely anatomical configuration of the concha, several surgeons have used the conchal bowl element, although the results are not published elsewhere. Dr. Nagata adopted the one-block or two-pieces conchal bowl element according to the amount of usable cartilages. However, the author believes that the reconstructed ear using his conchal bowl element shows a high crus helicis and step deformity in the concha. Therefore, the author modified Nagata's conchal bowl element so that it was carved extremely thinly at the conchal floor level and constructed a minimally sized cavum component. These modifications allow the reconstructed ear to show more natural contours in the concha area.

The conchal bowl element plays two roles. First, it stabilizes the crus helicis and achieves a realistic, smooth helical curve. Second, it converts the two-dimensional framework into a threedimensional structure.

The author constructed the conchal bowl element in one of three patterns according to the amount of available cartilages: one block, two-pieces, or a cymba bowl element only. Although the framework with the cymba bowl element only is less stable than the others, the aims of the conchal bowl element were achieved by it.

The construction of the conchal bowl element is a procedure that is neither time-consuming nor difficult. Thus, the author 
suggests that conchal bowl element be constructed and attached to the main framework for a natural configuration of the reconstructed ear.

\section{REFERENCES}

1. Nagata S. A new method of total reconstruction of the auricle for microtia. Plast Reconstr Surg 1993;92:187-201.

2. Nagata S. Modification of the stages in total reconstruction of the auricle: Part I. Grafting the three-dimensional costal cartilage framework for lobule-type microtia. Plast Reconstr Surg 1994;93:221-30.

3. Nagata S. Modification of the stages in total reconstruction of the auricle: Part II. Grafting the three-dimensional costal cartilage framework for concha-type microtia. Plast Reconstr Surg 1994;93:231-42.

4. Nagata S. Modification of the stages in total reconstruction of the auricle: Part III. Grafting the three-dimensional costal cartilage framework for small concha-type microtia. Plast Reconstr Surg 1994;93:243-53.

5. Nagata S. Modification of the stages in total reconstruction of the auricle: Part IV. Ear elevation for the constructed auricle. Plast Reconstr Surg 1994;93:254-66.

6. Kawanabe Y, Nagata S. A new method of costal cartilage harvest for total auricular reconstruction: part I. Avoidance and prevention of intraoperative and postoperative complications and problems. Plast Reconstr Surg 2006;117:2011-8.

7. Kawanabe Y, Nagata S. A new method of costal cartilage harvest for total auricular reconstruction: part II. Evaluation and analysis of the regenerated costal cartilage. Plast Reconstr Surg 2007;119:308-15.

8. Cho BC, Lee JH. New technique for the fabrication of a stable cartilage framework. Plast Reconstr Surg 2012;129: $183 \mathrm{e}-4 \mathrm{e}$.

9. Chin W, Zhang R, Zhang Q, et al. Modifications of threedimensional costal cartilage framework grafting in auricular reconstruction for microtia. Plast Reconstr Surg 2009;124: 1940-6.

10. Tanzer RC. Total reconstruction of the external ear. Plast Reconstr Surg Transplant Bull 1959;23:1-15.

11. Brent B. Auricular repair with autogenous rib cartilage grafts: two decades of experience with 600 cases. Plast Reconstr Surg 1992;90:355-74.

12. Brent B. Technical advances in ear reconstruction with autogenous rib cartilage grafts: personal experience with 1200 cases. Plast Reconstr Surg 1999;104:319-34.

13. Brent B. Microtia repair with rib cartilage grafts: a review of personal experience with 1000 cases. Clin Plast Surg 2002; 29:257-71.

14. Osorno G. A 20-year experience with the Brent technique of auricular reconstruction: pearls and pitfalls. Plast Reconstr Surg 2007; 119:1447-63.

15. Firmin F. Ear reconstruction in cases of typical microtia. Personal experience based on 352 microtic ear corrections. Scand J Plast Reconstr Surg Hand Surg 1998;32:35-47.

16. Tolleth $\mathrm{H}$. Artistic anatomy, dimensions, and proportions of the external ear. Clin Plast Surg 1978;5:337-45. 